\title{
CONTINUUM MODELING OF INTERFACES IN POLYMER MATRIX COMPOSITES REINFORCED BY CARBON NANOTUBES
}

\author{
LIYING JIANG $^{1^{*}}$, HONGLAI TAN ${ }^{2}$, JIAN WU ${ }^{3}$, YONGGANG HUANG ${ }^{2 *}$ AND KEH-CHIH HWANG \\ ${ }^{I}$ Department of Mechanical and Materials Engineering, The University of Western Ontario \\ London, Ontario N6A 5B9, Canada \\ ${ }^{2}$ Department of Mechanical Science and Engineering, University of Illinois at Urbana-Champaign, \\ Urbana, Illinois 61801, USA \\ ${ }^{3}$ Department of Engineering Mechanics, Tsinghua University, \\ Beijing 100084, China \\ 1*lyjiang@eng.uwo.ca, ${ }^{2 *}$ huang9@uiuc.edu
}

\begin{abstract}
The interface behavior may significantly influence the mechanical properties of carbon nanotube (CNT)-reinforced composites due to the large interface area per unit volume at the composite. The modeling of $\mathrm{CNT} /$ polymer interfaces has been a challenge in the continuum modeling of CNTreinforced composites. This paper presents a review of recent progress to model the CNT/matrix interfaces via a cohesive law established from the van der Waals force. A simple, analytical cohesive law is obtained from the interatomic potential, and is used to study the effect of CNT/matrix interfaces on the macroscopic properties of CNT-reinforced composites.
\end{abstract}

Keywords: Carbon nanotubes; cohesive law; interface; van der Waals force.

\section{Introduction}

Since the discovery of carbon nanotubes $(\mathrm{CNTs})^{1}$ and the establishment of new effective methods to produce them, ${ }^{2}$ the properties of these novel materials and their potential technological applications have stimulated considerable interests in the research and engineering communities. Due to their perfect molecular structure, CNTs are found to possess superior mechanical properties, such as high elastic modulus (on the order of $1 \mathrm{TPa})$, high tensile strength $(\sim 200 \mathrm{GPa})$ and high fracture strain $(10-30 \%),{ }^{3-12}$ and are therefore an ideal candidate for reinforcements in composite materials. ${ }^{13-}$ 18

One challenge in CNT-reinforced composites is the uniform dispersion and orientation alignment of CNTs in a matrix to avoid the agglomeration of CNTs into bundles. Significant efforts have been made to uniformly disperse and align CNTs in the matrix. ${ }^{19-23}$ For well-dispersed and aligned CNTs that are perfectly bonded to the matrix, the theoretical and computational models predict superior properties of CNT-reinforced composites. ${ }^{24-28}$ However, extensive experiments on CNT-reinforced composites ${ }^{20,29-34}$ show some improved properties, but many fall short to reach the theoretical predictions. The discrepancy between the theoretical models and experiments requires further investigations in order to fulfill the full potential of CNTs as reinforcement in composites.

Another challenge in CNT-reinforced composites is the load transfer efficiency across the CNT/matrix interface. Similar to the conventional fiber-reinforced composites, the interfacial load transfer between the 
CNTs and matrix is governed by three mechanisms. $^{27,29,35}$

(i) covalent bonding.

The covalent bonding between atoms from the CNT and matrix results from chemical reactions at the interface. The covalent bonding is strong. For example, Frankland et al. ${ }^{36}$ predicted that $\mathrm{CNT} /$ matrix shear strength can be enhanced by more than an order of magnitude with the formation of cross-links involving less than $1 \%$ of the carbon atoms on the CNT. The covalent bonding, however, requires the functionalization of CNT/matrix interfaces. This may increase the difficulty in processing, and also introduce defects to the $\mathrm{CNT},{ }^{34,37,38}$ which compromises the performance of composites. ${ }^{25,36,39}$ For example, the maximum compressive (buckling) force for ethyne functionalized nanotubes is reduced by $15 \%$ due to the functionalization. ${ }^{40}$

(ii) mechanical interlocking.

The mechanical interlocking usually results from defects around the interfaces in conventional composites. ${ }^{41-43}$ But this mechanism hardly occurs in CNTs because their (nearly) defect-free atomic structure. ${ }^{29}$

(iii) van der Waals force.

The van der Waals (vdW) force between CNTs and matrix is the most common mechanism for interfacial load transfer efficiency since it always exists and does not require any functionalization. The $\mathrm{vdW}$ force, however, is weak such that the CNTs do not bond well to the polymer matrix, which gives relatively low load transfer efficiency. ${ }^{27,29,33,44}$

There are extensive experimental and atomistic studies on the $\mathrm{vdW}$ force at the $\mathrm{CNT} /$ matrix interfaces. ${ }^{29-31,45-48}$ These studies provide insights into the fundamental understanding of CNT-matrix interactions, but not the direct relation between the $\mathrm{vdW}$ force and the macroscopic properties and behavior of CNT-reinforced composites. Furthermore, the atomistic studies widely used in studying the individual CNTs have limitations on both length scale $\left(10^{-9}-10^{-6} \mathrm{~m}\right)$ and time scale $\left(10^{-12}-10^{-9} \mathrm{~s}\right)$, and are not suitable to study macroscopic properties and behavior of CNT-reinforced composites, which involve large numbers of CNTs in the matrix.

Continuum models have been developed to study the mechanical properties of individual CNTs ${ }^{49-53}$ and also the CNT-reinforced composites. ${ }^{25-28,54}$ As compared to atomistic simulations such as molecular dynamics, the above continuum models for CNTreinforced composites are not constrained on the length and time scales, but they have not accounted for the important $\mathrm{vdW}$ force at the $\mathrm{CNT} /$ matrix interfaces. Since nanocomposites in general have high specific surface aspect ratio (i.e., high interface area per unit volume of the composite), the behavior of the $\mathrm{CNT} /$ matrix interfaces may significantly influence the macroscopic behavior of composites.

The interface debonding and sliding in conventional composites has been studied via cohesive zone models in the continuum analysis. ${ }^{55-57}$ A cohesive zone model assumes a relation between the normal (and shear) traction(s) and the opening (and sliding) displacement(s). When implemented in the finite element method, the cohesive zone model is capable of simulating interface debonding and sliding. ${ }^{58-68}$ The existing cohesive models, however, are all phenomenological because it is difficult to measure directly the cohesive laws for interfaces in experiments. There are some recent experimental studies of microscale cohesive laws, ${ }^{67,69-74}$ but none on nanoscale cohesive laws such as for the CNT/polymer interfaces. Recently, Jiang et al. $^{75}$ developed a cohesive law for CNT/polymer interfaces based on the $\mathrm{vdW}$ force. Such an approach avoids any assumed phenomenological cohesive laws, and accurately accounts for the vdW foces in the continuum model. Lu et al. ${ }^{76}$ extended such an approach to the cohesive law for multi-wall CNTs, and Tan et al. ${ }^{77}$ used this vdW-based cohesive law to study the effect of interface debonding on the macroscopic behavior of CNT-reinforced composites.

This paper provides a review of the continuum modeling of interfaces in CNT-reinforced polymer matrix composites. It focuses on the effect of $\mathrm{vdW}$ force on the CNT/matrix interface behavior and the macroscopic properties of CNT-reinforced composites. The interface cohesive law based on the vdW force is reviewed in Section 2, and its effect on the macroscopic behavior of CNT-reinforced polymer matrix composites is discussed in Section 3. 


\section{Cohesive Law for Carbon Nanotube/Polymer Interfaces}

The energy between two atoms of distance $r$ due to $\mathrm{vdW}$ interactions is usually represented by a pair potential $V(r)$. An example is the Lennard-Jones 6-12 potential,

$$
V(r)=4 \varepsilon\left(\frac{\sigma^{12}}{r^{12}}-\frac{\sigma^{6}}{r^{6}}\right),
$$

where $\sqrt[6]{2} \sigma$ is the equilibrium distance between the atoms and $\varepsilon$ is the bond energy at the equilibrium distance. For the vdW force between carbon atoms, $\sigma=0.341 \mathrm{~nm}$ and $\varepsilon=2.39 \mathrm{meV} .^{78}$ For the vdW force between a carbon atom on the $\mathrm{CNT}$ and a $-\mathrm{CH} 2-$ unit in the polyethylene, $\sigma=0.3825 \mathrm{~nm}$ and $\varepsilon=4.656 \mathrm{meV}^{47}$

\subsection{Cohesive law for graphene/polymer interfaces}

A graphene can be considered as a CNT with an infinitely large tube radius. The cohesive law between a graphene and a semi-infinite polymer matrix can be obtained analytically from the vdW interactions. ${ }^{75}$ Let $h$ denote the equilibrium spacing between a graphene and a semi-infinite polymer matrix (Fig. 1a). The carbon atoms on the graphene are represented by their area density $\rho_{c}=\frac{4}{3 \sqrt{3} l_{0}^{2}}$, where $l_{0}$ is the equilibrium bond length. The number of carbon atoms over area $d A$ on the graphene is $\rho_{c} d A$. Similarly the volume density of polymer molecules is denoted by $\rho_{p}$, and the number of polymer molecules over a volume $d V$ is $\rho_{p} d V$. The energy stored in an infinitesimal area $d A$ due to the vdW force is $\rho_{c} d A \int_{V_{\text {polymer }}} V(r) \rho_{p} d V_{\text {polymer }}$, where $V(r)$ can be an arbitrary pair potential, and the integration is over the polymer volume. The cohesive energy $\Phi$ is the energy stored per unit area, and is given by

$$
\Phi=2 \pi \rho_{p} \rho_{c} \int_{h}^{\infty} V(r) r(r-h) d r
$$

where the integration over the polymer volume is carried out analytically, and $h$ is the equilibrium distance between the graphene and polymer (Fig. 1a) that is determined from energy minimization $\frac{\partial \Phi}{\partial h}=0$ by

$$
\int_{h}^{\infty} V(r) r d r=0 .
$$

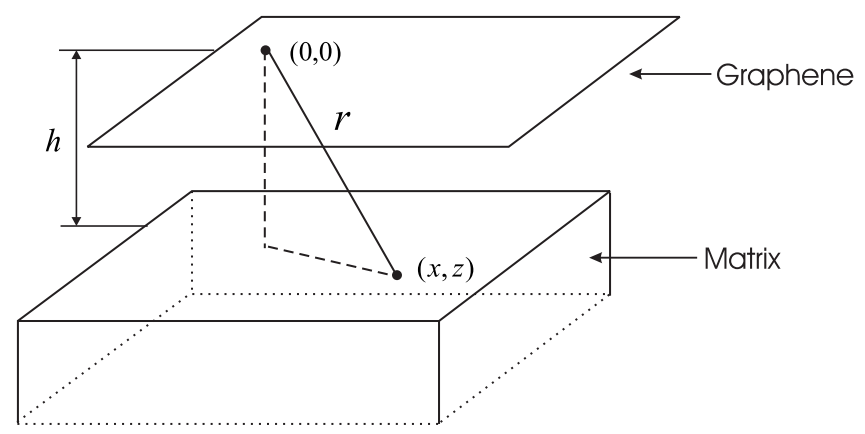

Fig. 1a. A schematic diagram of a graphene parallel to the surface of a polymer and the distance between graphene and polymer surface is $h$

Once the graphene is subjected to an opening displacement $v$ beyond the equilibrium distance $h$ as shown in Fig. 1b, the distance between the graphene and polymer becomes $h+v$. The cohesive energy is then obtained from Eq. (2) by simply replacing $h$ with $h+v$,

$$
\Phi(v)=2 \pi \rho_{p} \rho_{c} \int_{h+v}^{\infty} V(r) r(r-h-v) d r .
$$

The tensile cohesive stress is then obtained as

$$
\sigma^{\mathrm{int}}(v)=\frac{\partial \Phi}{\partial v}=-2 \pi \rho_{p} \rho_{c} \int_{h+v}^{\infty} V(r) r d r .
$$

Since the cohesive energy in Eq. (4) is independent of the sliding displacement $u$, it gives a vanishing shear cohesive stress

$$
\tau^{\mathrm{int}}=\frac{\partial \Phi}{\partial u}=0 .
$$

The vanishing shear cohesive stress above is the consequence of average van der Waals interactions at the interface. For CNT composites with interfacial functionalization and/or mechanical interlocking, the shear cohesive stress may not vanish any more, just as in fiber-reinforced composites. 
The equilibrium distance, cohesive energy, and cohesive stresses in Eqs. (3)-(6) hold for an arbitrary pair potential $V(r)$ of the vdW force. For the Lennard Jones 6-12 potential in Eq. (1), the equilibrium distance in Eq. (3) is given by

$$
h=(2 / 5)^{1 / 6} \sigma=0.858 \sigma .
$$

The tensile cohesive stress in Eq. (5) then becomes

$$
\begin{gathered}
\sigma^{\mathrm{int}}(v)=(50)^{1 / 3} \pi \rho_{p} \rho_{c} \varepsilon \sigma^{2}\left\{\left[1+\frac{v}{(2 / 5)^{1 / 6} \sigma}\right]^{-4}\right. \\
\left.-\left[1+\frac{v}{(2 / 5)^{1 / 6} \sigma}\right]^{-10}\right\} .
\end{gathered}
$$

which is the cohesive law for graphene/polymer interface. It gives the total cohesive energy (area under $\sigma^{\text {int }} \sim v$ curve) $\Phi_{\text {total }}=\frac{4 \pi}{9} \sqrt{\frac{5}{2}} \rho_{p} \rho_{c} \varepsilon \sigma^{3}$, and cohesive strength (maximum cohesive stress) $\sigma_{\max }=\frac{6 \pi}{5} \rho_{p} \rho_{c} \varepsilon \sigma^{2}$ at the opening displacement $v=\delta_{0}=\left[1-(2 / 5)^{1 / 6}\right] \sigma . \quad$ For $\mathrm{CNT} /$ polyethylene, this critical separation becomes $\delta_{0}=0.0542 \mathrm{~nm}$.

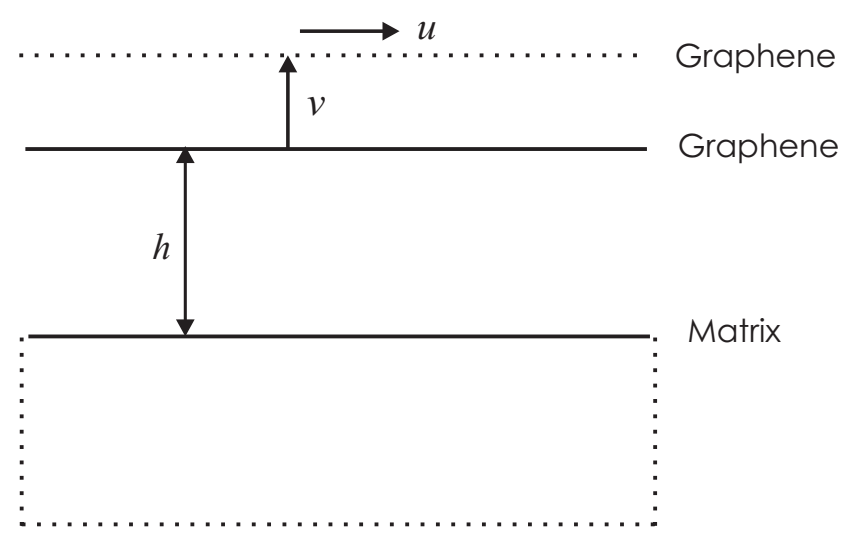

Fig. 1b. A schematic diagram of a graphene parallel to the surface of a polymer; the graphene is subjected to the opening and sliding displacements.

Figure 2 shows the tensile cohesive stress $\sigma^{\text {int }}$, normalized by the cohesive strength $\sigma_{\max }$, versus the normalized opening displacement $\mathrm{v} / \delta_{0}$. The cohesive stress increases rapidly at small opening displacement, reaches the cohesive strength, and gradually decreases after the peak is reached.

\subsection{Cohesive law for carbon nanotube/polymer interfaces}

Figure 3 shows a CNT embedded in a polymer matrix

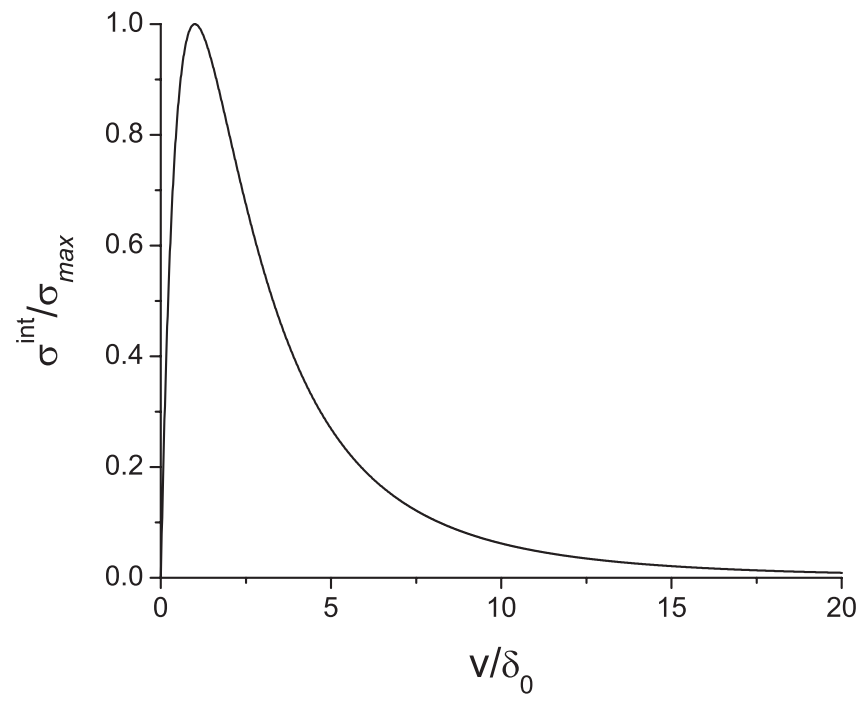

Fig. 2. The cohesive law for a graphene and polymer matrix established from the van der Waals interactions, where $\sigma_{\max }$ is the cohesive strength and $\delta_{0}$ is the critical separation at which the cohesive strength is reached.

with the equilibrium spacing $h$. For a section of CNT and polymer of height $d z$, the energy due to the van der Waals force is $\rho_{c} 2 \pi R d z \int_{V_{\text {polvmer }}} V(r) \rho_{p} d V_{\text {polymer }}$.

The cohesive energy $\Phi$ is the energy per unit area, and is given by

$$
\begin{gathered}
\Phi=\frac{2 \pi \rho_{p} \rho_{c} R d z \int_{V_{\text {polymer }}} V(r) d V_{\text {polymer }}}{2 \pi\left(R+\frac{h}{2}\right) d z} \\
=\frac{\rho_{p} \rho_{c} \int_{V_{\text {polymer }}} V(r) d V_{\text {polymer }}}{1+\frac{h}{2 R}},
\end{gathered}
$$

where $2 \pi\left(R+\frac{h}{2}\right) d z$ is the average of CNT area $2 \pi R d z$ and polymer surface area $2 \pi(R+h) d z$. For the Lennard-Jones potential in (1), the cohesive energy above becomes ${ }^{75}$ 
$\Phi=\pi \rho_{p} \rho_{c} \varepsilon \sigma^{3} \frac{R}{2 R+h} \int_{0}^{2 \pi}\left(\sigma^{9} \varphi_{9}-\sigma^{3} \varphi_{3}\right) d \theta$,

where

$$
\begin{aligned}
& \varphi_{9}=\frac{7}{32 R_{h}^{9}}+\frac{R \cos \theta}{R_{h}^{9}\left(R_{h}+R_{h}^{\prime}\right)^{5}}\left(\frac{4}{5} R_{h}^{4}+\frac{65}{32} R_{h}^{3} R_{h}^{\prime}\right. \\
& \left.+\frac{69}{32} R_{h}^{2} R_{h}^{\prime 2}+\frac{35}{32} R_{h} R_{h}^{\prime 3}+\frac{7}{32} R_{h}^{\prime 4}\right), \\
& \varphi_{3}=\frac{1}{R_{h}^{3}}+\frac{R \cos \theta}{R_{h}^{3}\left(R_{h}+R_{h}^{\prime}\right)^{2}}\left(2 R_{h}+R_{h}^{\prime}\right), \\
& R_{h}=\sqrt{(R+h)^{2}+R^{2}-2 R(R+h) \cos \theta}
\end{aligned}
$$

and $R_{h}^{\prime}=R+h-R \cos \theta$.

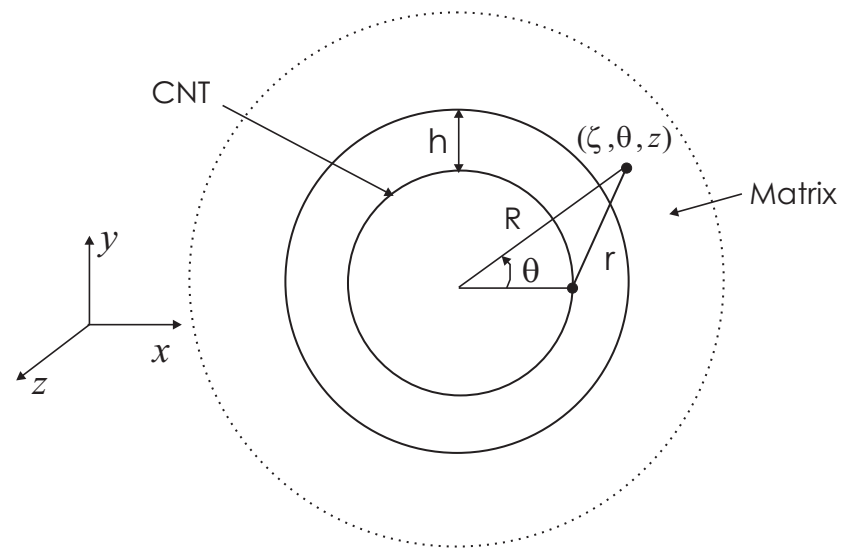

Fig. 3. A schematic diagram of a carbon nanotube $(\mathrm{CNT})$ in a polymer matrix.

Figure 4 shows the cohesive energy in (10), normalized by the total cohesive energy for graphene $\Phi_{\text {total }}=\frac{4 \pi}{9} \sqrt{\frac{5}{2}} \rho_{p} \rho_{c} \varepsilon \sigma^{3}$, versus the distance $h$ between the CNT and polymer surface for several CNT radii, where $h$ is normalized by its equilibrium value $(2 / 5)^{1 / 6} \sigma$ in (7) for graphehe, and $\sigma=0.3825$ $\mathrm{nm}$ is the characteristic length in the van der Waals force. The curves for different CNTs are very close, and they are all close to that for graphene. This suggests that the CNT radius has little effect on the cohesive energy. Each curve has a minimum, corresponding to the equilibrium distance between the $\mathrm{CNT}$ and polymer surface, and this distance is very close to $(2 / 5)^{1 / 6} \sigma$.
For CNTs embedded in a polyethylene matrix, the area density of carbon atoms on the CNT can be estimated by that for the graphene, $\rho_{c}=\frac{4}{3 \sqrt{3} l_{0}^{2}}=3.82 \times 10^{19} \mathrm{~m}^{-2}$. The volume density of polymer molecules is $\rho_{p}=3.1 \times 10^{28} \mathrm{~m}^{-3}$, which is determined by polyethylene mass density $0.71 \times 10^{3}$ $\mathrm{kg} \cdot \mathrm{m}^{-3}$ (Frankland et al. ${ }^{47}$ ) and the mass of $-\mathrm{CH} 2-$ unit $\left(2.3 \times 10^{-26} \mathrm{~kg}\right)$. These, together with $\sigma=0.3825 \mathrm{~nm}$ and $\varepsilon=4.656 \mathrm{meV}$ for the $\mathrm{vdW}$ force between a carbon atom on the $\mathrm{CNT}$ and a $-\mathrm{CH} 2-$ unit in the polyethylene, ${ }^{47}$ give a rather high interfacial cohesive strength $\sigma_{\text {max }}=479 \mathrm{MPa}$ as compared to the Young's modulus $(\sim 0.9 \mathrm{GPa})$ of the polyethylene matrix. However, the total cohesive energy $\Phi_{\text {total }}=0.107 \mathrm{~J} / \mathrm{m}^{2}$ is low, which is consistent with the poor bonding between CNTs and matrix.

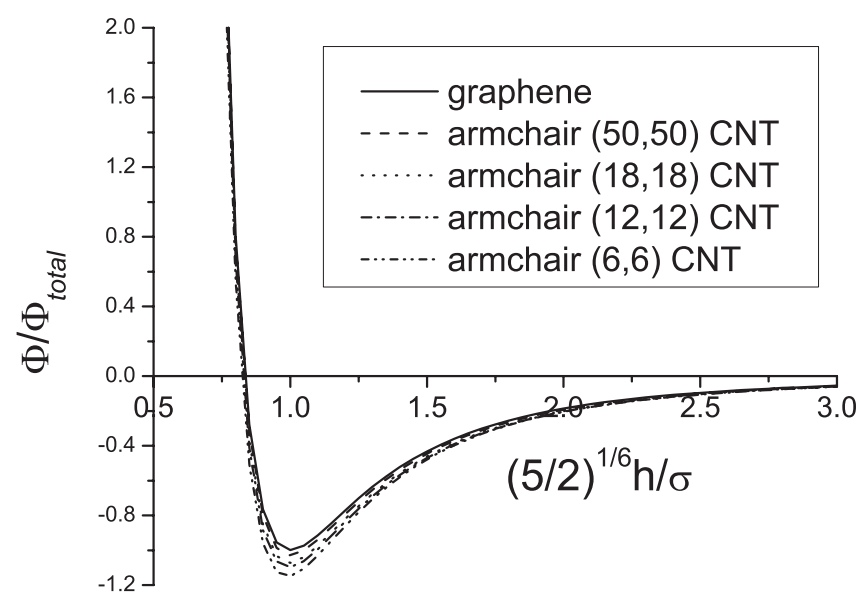

Fig. 4. The cohesive energy, normalized by the total cohesive energy $\Phi_{\text {total }}$ for graphene, versus the normalized distance $h$ between the carbon nanotube (CNT) and polymer surface for several CNT radii and graphene, where $\mathrm{h}$ is normalized by its equilibrium value $(2 / 5)^{1 / 6} \sigma$ for graphene.

\subsection{Cohesive law accounting for polymer surface roughness}

Polymer of chain molecules exhibits irregular surface structure and roughness, ${ }^{37,79}$ and may not have the flat surface assumed in Sections 2.1 and 2.2. Figure 5 shows a wavy polymer surface with amplitude $\Delta$ and wavelength $\lambda$. The average distance between the polymer surface and graphene is still denoted by $h$. The average cohesive energy for an arbitrary pair potential $V(r)$ is given by 


$$
\Phi=\rho_{p} \rho_{c} \frac{1}{\lambda} \int_{-\lambda / 2}^{\lambda / 2} d z_{c} \int_{V_{\text {polymer }}} V(r) d V_{\text {polymer }}
$$

where $\frac{1}{\lambda} \int_{-\lambda / 2}^{\lambda / 2} d z_{c}$ represents the average over the wavelength $\lambda$. For the opening displacement $v$ and sliding displacement $u$ (along the wavy direction $x$ ) as shown in Fig. 5, the cohesive energy can be similarly obtained.

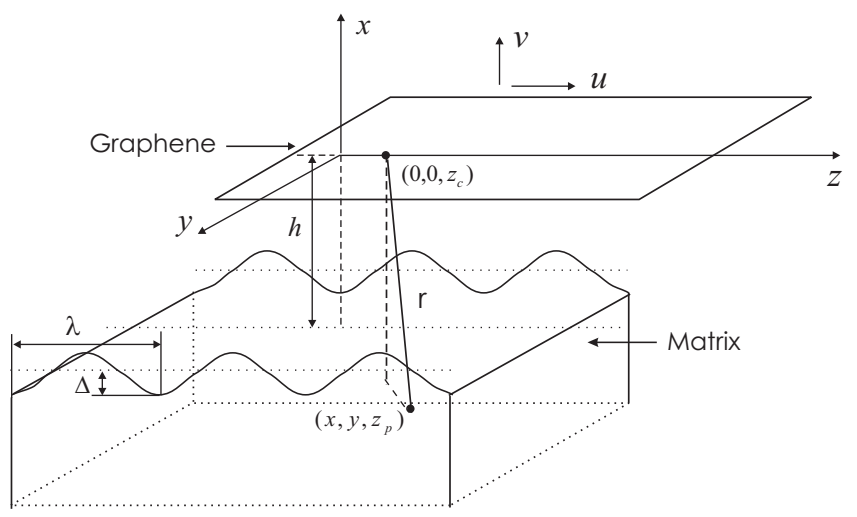

Fig. 5. A schematic diagram of a graphene parallel to the wavy surface of polymer matrix.

The tensile cohesive stresses $\sigma^{\text {int }}=\frac{\partial \Phi}{\partial v}$ can be analytically obtained as ${ }^{75}$

$$
\begin{gathered}
\sigma^{\mathrm{int}}=-2 \pi \rho_{p} \rho_{c}\left[\int_{h+v-\Delta}^{\infty} V(r) r d r\right. \\
\left.-\frac{1}{\pi} \int_{h+v-\Delta}^{h+v+\Delta} V(r d) r \cos ^{-1} \frac{r-h-v}{\Delta} d r\right] .
\end{gathered}
$$

Since the amplitude $\Delta$ of polymer wavy surface must be less than $h+v$ (otherwise the polymer surface penetrates the graphene), the Taylor expansion of Eq. (12) with respect to $\Delta$ gives

$$
\begin{gathered}
\sigma^{\mathrm{int}}=-2 \pi \rho_{p} \rho_{c} \int_{h+v}^{\infty} V(r) r d r+\frac{\pi}{2} \rho_{p} \rho_{c} \\
{\left[V(h+V)+(h+v) V^{\prime}(h+v)\right] \Delta^{2}+O\left(\Delta^{3}\right) .}
\end{gathered}
$$

Its difference with the tensile cohesive stress in (5) for a flat polymer surface is on the order of $\Delta^{2}$. Figure 6 shows the tensile cohesive stress given by Eq. (12) (not the Taylor series expansion (13)) versus the normalized opening displacement $\mathrm{v} / \delta_{0}$ for several waviness amplitudes $\Delta=0,0.2,0.4,0.6$ and $0.8 h$, where $\Delta=0$ corresponds to no waviness and $h$ is equilibrium spacing. The tensile cohesive stress $\sigma^{\text {int }}$ is normalized by the cohesive strength $\sigma_{\max }$ without waviness. As the waviness $\Delta$ increases, both the cohesive strength (peak value of the curve) and total cohesive energy (area underneath the curve) decrease. For example, the cohesive strength drops from $\sigma_{\max }$ for $\Delta=0$ to $0.81 \sigma_{\max }$ for $\Delta=0.2 h, 0.60 \sigma_{\max }$ for $\Delta=0.4 h, \quad 0.48 \sigma_{\max }$ for $\Delta=0.6 h$ and $0.41 \sigma_{\max }$ for $\Delta=0.8 h$, respectively.

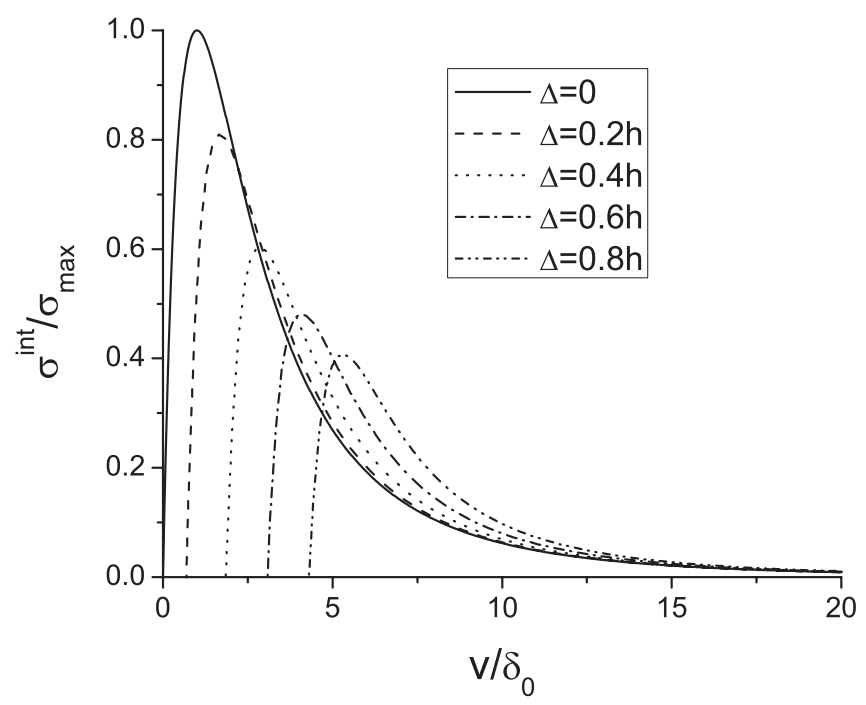

Fig. 6. The cohesive law for CNT/polymer matrix with different polymer surface waviness $\Delta$, where $\mathrm{h}$ is the equilibrium spacing, $\sigma_{\max }$ is the cohesive strength, and $\delta_{0}$ is the critical separation at which the cohesive strength is reached.

The average shear cohesive stress also vanishes $\tau^{\mathrm{int}}=\frac{\partial \Phi}{\partial u}=0 .^{75}$ This represents shear stress averaged over the period of wavy polymer surface. However, the local shear stress may not be zero.

\section{Macroscopic Behavior of Nanocomposites Accounting for the Interface Cohesive Law}

Tan et al. ${ }^{77}$ developed a theoretical framework to account for the effect of interfaces in CNT-reinforced polymer matrix composites. The CNTs are modeled as elastic fibers, with the Young's modulus and 
Poisson's ratio determined from the interatomic potential. ${ }^{52}$ The fiber (CNT) volume fraction is $f$.

The macroscopic stress $\bar{\sigma}$ and strain $\bar{\varepsilon}$ of the CNT-reinforced nanocomposites represent the collective, homogenized behavior of fibers (CNTs) and matrix. They can be expressed in terms of the average stress $\sigma$ and strain $\varepsilon$ in the fibers and matrix as

$$
\begin{gathered}
\bar{\sigma}=f \sigma^{f}+(1-f) \sigma^{m}, \\
\bar{\varepsilon}=f \varepsilon^{f}+(1-f) \varepsilon^{m}+f \varepsilon^{\mathrm{int}},
\end{gathered}
$$

where the superscripts $f$ and $m$ denote the fibers (CNTs) and matrix, respectively. The additional term $f \varepsilon^{\text {int }}$ represents the contribution from the $\mathrm{CNT} /$ matrix interfaces, which is related to the displacement discontinuity $[\mathbf{u}]=\mathbf{u}^{m}-\mathbf{u}^{f}$ across the CNT/matrix interface by

$$
\boldsymbol{\varepsilon}^{\mathrm{int}}=\frac{1}{2 V^{f}} \int_{S^{\text {int }}}([\mathbf{u}] \otimes \mathbf{n}+\mathbf{n} \otimes[\mathbf{u}]) d A .
$$

Here $S^{\text {int }}$ represents CNT/matrix interface area and $V^{f}$ is the fiber volume.

The matrix and CNTs are linear elastic with elastic compliance tensor $\mathbf{M}^{m}$ and $\mathbf{M}^{f}$, where $\mathbf{M}^{f}$ is determined from the interatomic potential. ${ }^{52}$ The stresses and strains in the matrix and CNTs satisfy their constitutive laws, respectively,

$$
\boldsymbol{\varepsilon}^{m}=\mathbf{M}^{m}: \boldsymbol{\sigma}^{m}, \quad \boldsymbol{\varepsilon}^{f}=\mathbf{M}^{f}: \boldsymbol{\sigma}^{f} .
$$

The macroscopic strain $\overline{\boldsymbol{\varepsilon}}$ of the composite can be obtained in terms of the macroscopic stress $\overline{\boldsymbol{\sigma}}$ via Eqs. (14), (15) and (17) as

$$
\overline{\boldsymbol{\varepsilon}}=\mathbf{M}^{m}: \overline{\boldsymbol{\sigma}}+f\left\{\left(\mathbf{M}^{f}-\mathbf{M}^{m}\right): \boldsymbol{\sigma}^{f}+\boldsymbol{\varepsilon}^{\text {int }}\right\},
$$

where $\varepsilon^{\text {int }}$ and $\sigma^{f}$ are to be determined from the interface cohesive law, as illustrated in the following for an isotropic matrix containing randomly distributed, long and isotropic fibers subjected to hydrostatic tension $\overline{\boldsymbol{T}}$, and $\boldsymbol{I}$ is the second-order identity tensor. The macroscopic strain of the composite is $\bar{\varepsilon} \boldsymbol{I}$, and $\bar{\varepsilon}$ is obtained from Eq. (18) as

$$
\bar{\varepsilon}=\frac{1}{3 K_{m}} \bar{\sigma}+\frac{f}{3}\left[\left(\frac{1}{3 K_{f}}-\frac{1}{3 K_{m}}\right) \sigma_{k k}^{f}+\varepsilon_{k k}^{\mathrm{int}}\right],
$$

where $K_{m}$ and $K_{f}$ are the elastic bulk moduli of the matrix and fibers, respectively, $\varepsilon_{k k}^{\text {int }}$ is related to the displacement jump $[v]$ in the normal direction across the fiber/matrix interface via (16) by

$$
\varepsilon_{k k}^{\mathrm{int}}=2 \frac{[v]}{R},
$$

and $R$ is the CNT radius.

Since the CNT volume fraction is usually low, the dilute model, which accounts for the CNT/matrix interactions but neglects the interactions among CNTs, can be used to determine $\sigma_{k k}^{f}$ and $\varepsilon_{k k}^{\text {int }}$ in terms of $\bar{\sigma}$. Let $\bar{\sigma}$ and $\bar{\varepsilon}$ denote the macroscopic stress and strain of the composite, and $\sigma^{\text {int }}$ is the normal cohesive stress at the fiber/matrix interface. In conjunction with the cohesive law $\sigma^{\text {int }}=\sigma^{\text {int }}([v])$ in Eq. (8), the dilute model gives the macroscopic stress $\bar{\sigma}$ in terms of $[v]^{77}$ as

$$
\bar{\sigma}=\frac{\frac{[v]}{R}+\left(\frac{1+v_{m}}{E_{m}}+\frac{1-v_{f}}{E_{f}}\right) \sigma^{\mathrm{int}}([v])}{\frac{2-v_{m}}{E_{m}}+\frac{v_{f}}{E_{f}}},
$$

where $E_{m}, v_{m}, E_{f}$ and $v_{f}$ are the Young's modulus and Poisson's ratio of the matrix and fibers, respectively. The macroscopic strain in Eq. (19) is given in terms of $[v]^{77}$ as

$$
\bar{\varepsilon}=\frac{1}{3 K_{m}} \bar{\sigma}+\frac{f}{3}\left\{\left(\frac{1}{3 K_{f}}-\frac{1}{3 K_{m}}\right)\left[2 \sigma^{\mathrm{int}}([v])+\bar{\sigma}\right]+2 \frac{[v]}{R}\right\}
$$

Eqs. (21) and (22) give the macroscopic stress $\bar{\sigma}$ and strain $\bar{\varepsilon}$ in terms of $[v]$, and therefore provide the nonlinear stress-strain relation of the CNT-reinforced composite.

The CNT Young's modulus $E_{f}$ is on the order of $1 \mathrm{TPa},{ }^{52}$ which is several orders of magnitude higher than the modulus $E_{m}$ of polymer matrix (e.g., $0.9 \mathrm{GPa}$ for polyethylene). There are variations of the CNT Young's modulus, ${ }^{80}$ but they are all much higher than the modulus of polymer. For a solid rod model in nanocomposites ${ }^{54}$ the modulus of the effective fiber is also much higher than that of the polymer matrix. Equations (21) and (22) can therefore be simplified to 


$$
\begin{gathered}
\bar{\sigma}=\frac{E_{m}}{2-v_{m}} \frac{[v]}{R}+\frac{1+v_{m}}{2-v_{m}} \sigma^{\mathrm{int}}([v]), \\
\bar{\varepsilon}=\frac{1-2 v_{m}+f}{2-v_{m}} \frac{[v]}{R} \\
+\frac{1}{E_{m}}\left[1+v_{m}-\frac{f}{3}\left(5-v_{m}\right)\right] \frac{1-2 v_{m}}{2-v_{m}} \sigma^{\text {int }}([v]),
\end{gathered}
$$

which are independent of the CNT Young's modulus. For each given $[v]$, the above two equations give the macroscopic stress and strain, and therefore the nonlinear stress-strain relation of the CNT-reinforced composites.

Figure 7 shows the stress-strain curve for a CNTreinforced polyethylene composite subjected to hydrostatic tension. The Young's modulus and Poisson's ratio of the polyethylene matrix are $E_{m}=0.9 \mathrm{GPa}$ and $v_{m}=0.3$, respectively. ${ }^{47}$ The CNTs are armchair $(18,18)$, with the radius $R=1.25$ $\mathrm{nm}$, and the volume fraction is $f=1 \%, 5 \%$ and $10 \%$. The interface cohesive law in (8) is used for the $\mathrm{CNT}$ /polyethylene matrix, with $\rho_{c}=\frac{4}{3 \sqrt{3} l_{0}^{2}}=3.82 \times 10^{19} \mathrm{~m}^{-2}, \quad \rho_{p}=3.1 \times 10^{28} \mathrm{~m}^{-3}$, $\sigma=0.3825 \mathrm{~nm}$ and $\varepsilon=4.656 \mathrm{meV}$. The stress-strain curve for $f=10 \%$ has a peak strength $406 \mathrm{MPa}$, at which the strain is $13.9 \%$. Both the stress and strain decrease after the peak strength is reached due to the softening behavior in the $\mathrm{CNT} /$ matrix interface cohesive law. The CNT/matrix interfaces reach complete debonding when the strain drops to about $9.1 \%$, after which the stress and strain start to increase again due to the linear elastic behavior of the matrix. For the displacement-controlled experiment, the stressstrain curve would drop vertically from $406 \mathrm{MPa}$ to $289 \mathrm{MPa}$ at the $13.9 \%$ strain, and then gradually increase. For $\mathrm{f}=1 \%, 5 \%$ and $10 \%$, the peak strength is exactly the same, $406 \mathrm{MPa}$, such that the CNT volume fraction has no effect on the composite strength. But the critical strain at the peak strength changes slightly, $13.9 \%$ for $\mathrm{f}=10 \%, 14.6 \%$ for $\mathrm{f}=5 \%$, and $15.2 \%$ for $\mathrm{f}=1 \%$. Therefore the higher volume fraction of CNT gives higher initial modulus but gives lower incremental modulus at larger strain due to the debonding of CNTs. Therefore, CNTs may reinforce the composite at the small strain but weaken it at the large strain if there is only the vdW force at the $\mathrm{CNT} /$ matrix interfaces.

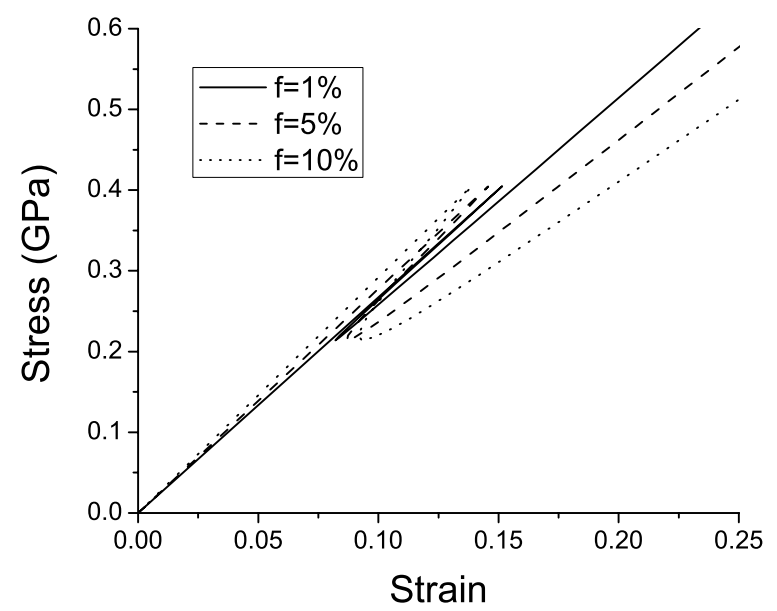

Fig. 7. The macroscopic stress-strain relation of a carbon nanotube-reinforced polyethylene matrix composite with varying volume fraction and CNTs are armchair $(18,18)$.

Figure 8 shows the effect of CNT size on the stress-strain curve with CNT volume fraction being fixed at $\mathrm{f}=10 \%$. Three different armchair CNTs, $(18,18),(12,12)$ and $(6,6)$, are examined, and the corresponding radii are $\mathrm{R}=1.25,0.83$ and $0.42 \mathrm{~nm}$, respectively. The CNT radius has a significant effect on the nanocomposites behavior since the peak strength increases with the decrease of CNT radius. Small CNTs clearly give stronger reinforcing effect than large CNTs because, at a fixed CNT volume fraction, there are more small CNTs than large ones, and therefore more interfaces. This observation of strong reinforcing effect for small CNTs also holds after CNTs are debonded from the matrix. Therefore, small CNTs are more effective than large ones.

\section{Summary}

This paper provides a review of the continuum modeling of carbon nanotube (CNT)/polymer interfaces based on van der Waals force, and the effect of interfaces on the macroscopic properties of CNTreinforced polymer matrix composites. The continuum cohesive law for CNT/polymer interfaces is obtained analytically from the Lennard-Jones potential, as well as from any interatomic potential, for the van der Waals force. These critical parameters in the cohesive law, namely the cohesive strength and 
total cohesive energy, are given analytically in terms of the area density of carbon atoms on the nanotube and volume density of polymer molecules, as well as the parameters in the van der Waals force. For the $\mathrm{CNT} /$ polyethylene matrix, the cohesive strength is very large, $479 \mathrm{MPa}$, but the total cohesive energy is small, $0.107 \mathrm{~J} / \mathrm{m}^{2}$, which reflects the relatively weak interactions of the vdW force.

A micromechanics model has been developed to study the macroscopic behavior for CNT-reinforced composites accounting for the CNT/polymer interfaces. It is shown that CNTs can indeed improve the mechanical properties of the composite at small strain. However, such improvement disappears at relatively large strain due to debonding of (or vanishing interactions at) $\mathrm{CNT} /$ polymer interfaces.

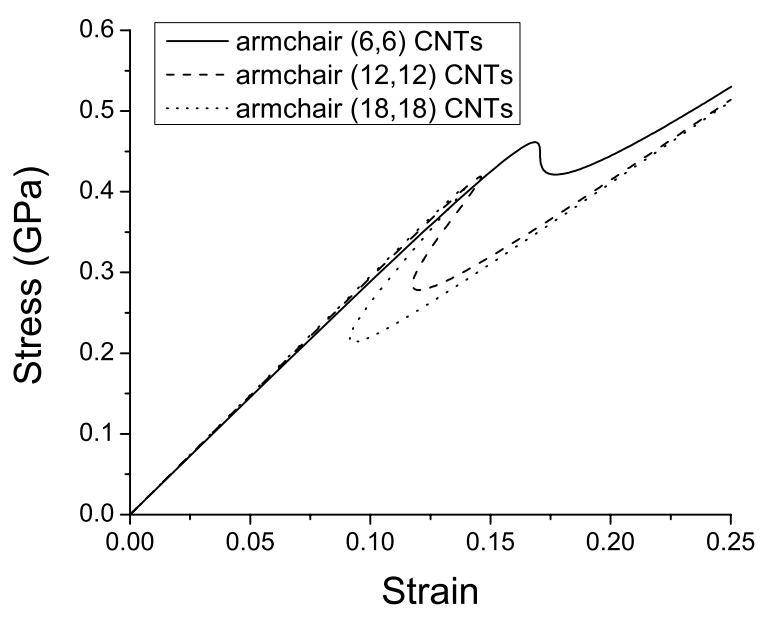

Fig. 8. The macroscopic stress-strain relation of a carbon nanotube-reinforced polyethylene matrix composite with varying sizes and fixed volume fraction $\mathrm{f}=10 \%$.

\section{Acknowledgements}

L. Y. Jiang was supported by the Natural Sciences and Engineering Research Council of Canada (NSERC). H. Tan and Y. Huang were supported by ONR Composites for Marine Structures Program (grant N00014-01-1-0205, Program Manager Dr. Y. D. S. Rajapakse), NSF and NSFC.

\section{References}

1. S. Iijima, Nature 354, 56 (1991).
2. A. Thess, R. Lee, P. Nikolaev, H. J. Dai, P. Petit, J. Roert, C. H. Xu, Y. H. Lee, S. G. Kim, A. G. Rinzler, D. T. Colbert, G. E. Scuseria, D. Tomanek, J. E. Fischer and R. E. Smalley, Science 273, 483 (1996).

3. M. M. J. Treacy, T. W. Ebbesen, J. M. Gibson, Nature 381, 678 (1996).

4. B. I. Yakobson, C. J. Brabec and J. Bernholc, Phys. Rev. Letts. 76, 2511 (1996).

5. B. I. Yakobson, M. P. Campbell, C. J. Brabec and J. Bernholc, Comp. Mater. Sci. 8, 341 (1997).

6. A. Krishnan, E. Dujardin, T. W. Ebbesen, P. N. Yianilos and M. M. J. Treacy, Phys. Rev. B 58, 14013 (1998).

7. E. W. Wong, P. E. Sheehan and C. M. Lieber, Science 277, 1971 (1997).

8. J. P. Salvetat, G. A. D. Briggs, J. M. Bonard, R. R. Bacsa, A. J. Kulik, T. Stockli, N. A. Burnham and L. Forro, Phys. Rev. letts. 82, 944 (1999).

9. M. F. Yu, B. S. Files, S. Arepalli and R. S. Ruoff, Phys. Rev. Letts. 84, 5552 (2000)

10. M. F. Yu, O. Lourie, M. J. Dyer, K. Moloni, T. F. Kelly and R. S. Ruoff, Science 287, 637 (2000).

11. C. F. Cornwell and L. T. Wille, Solid. Stat. Comm. 101, 555 (1997).

12. V. N. Popov, V. E. Van Dore and M. Balkanski, Phys. Rev. B 61, 3078 (2000).

13. E. T. Thostenson, Z. F. Ren and T. W. Chou, Compos. Sci. Technol. 61, 1899 (2001).

14. E. T. Thostenson, C. Y. Li and T. W. Chou, Compos. Sci. Technol. 65, 491 (2005).

15. B. Maruyama and H. Alam, SAMPE J. 38, 59 (2002).

16. S. Deepak, C. Wei and K. Cho, Appl. Mech. Rev. 56, 215 (2003).

17. O. Breuer and U. Sundararaj, Polym. Compos. 25, 630 (2004).

18. P. J. F. Harris, Int. Mater. Rev. 49, 31 (2004).

19. C. Park, Z. Ounaies, K. A.Watson, R. E. Crooks and J. Smith, Chem. Phys. Letts. 364, 303 (2000).

20. X. Y. Gong, J. Liu, S. Baskaran, R. D.Voise and J. S. Young, Chem. of Mater. 12, 1049 (2000).

21. C. A. Cooper, D. Ravich, D. Lips, J. Mayer and H. D.Wagner, Compos. Sci. Technol. 62, 1105 (2002).

22. R. Haggenmueller, H. H. Gommans, A. G. Rinzler, J. E. Fische and K. I. Winey, Chem. Phys. Letts. 330, 219 (2000).

23. A. Dufresne, M. Paillet, J. L. Putaux, R. Canet, F. Carmona, P. Delhaes and S. Cui, J. Maters. Sci. 37, 3915 (2002).

24. S. B. Sinnott, O. A. Shenderova, C. T. White and D. W. Brenner. Carbon, 36, 1 (1998).

25. G. M. Odegard, T. S. Gates, K. E., Wise, C. Park and E. J. Siochi, Compos. Sci. Technol. 63,1671 (2003)

26. Y. J. Liu and X. L. Chen, Mech. Mater. 35, 69 (2003).

27. C. Y. Li and T. W. Chou, J. Nanosci. Nanotechno. 3, 423 (2003).

28. D. L. Shi, X. Q. Feng, Y. Huang, K. C. Hwang and H. Gao, J. Eng. Mater. Technol. 126, 250 (2004).

29. L. S. Schadler, S. C. Giannaris and P. M. Ajayan, Appl. Phys. Lett. 73, 3842 (1998).

30. H. D. Wagnger, O. Lourie, Y. Feldman and R. Tenne, Appl. Phys. Letts. 72,188 (1998). 
31. D. Qian, E. C. Dickey, R. Andrews and T. Rantell, Appl. Phys. Letts. 76, 2868 (2000).

32. R. Andrews, D. Jacques, A. M. Rao, T. Rantell, F. Derbyshire, Y. Chen, J. Chen and R. C. Haddon, Appl. Phys. Letts. 75, 1329 (1999).

33. P. M. Ajayan, L. S. Schadler, C. Giannaris and A. Rubio, Advanced Maters. 12, 750 (2000).

34. K. T. Lau, S. Q. Shi and H. M. Cheng, Compos. Sci. Technol. 63, 1161 (2003).

35. A. V. Desai and M. A. Haque, Thin-Walled Struct. 43, 1787 (2005)

36. S. J. V. Frankland, A. Caglar, D. W. Brenner and M. Griebel, J. Phys. Chem. B 106, 3046 (2002).

37. Y. Lordi and N. Yao, J. Maters. Res. 15, 2770 (2000).

38. R. J. Chen, Y. Zhang, D. Wang and H. Dai, J. Am. Chem. Soc. 123, 3838 (2001).

39. A. Eitan, K. Y. Jiang, D. Dukes, R. Andrews and L. Schadler, Chem. Mater. 15, 3198 (2003).

40. A. Garg and S. B. Sinnott, Chem. Phys. Lett. 295, 273 (1998)

41. Y. L. Huang and R. G. Young, Coms. Sci. Technol. 52, 505 (1994).

42. H. F. Wang, J. C. Nelson, C. L. Lin and W.W. erberich, J. Mater. Res. 9, 498 (1994).

43. S. I. Moon and J. Jang, J. Maters. Sci. 34, 4219 (1999).

44. K. T. Lau and S. Q. Shi, Carbon 40, 2965 (2002).

45. K. Liao and S. Li, Appl. Phys. Letts. 79, 4225 (2001).

46. M. Wong, M. Paramsothy, X. J. Xu, Y. Ren, S. Li and K. Liao, Polymer 44, 7757 (2003).

47. S. J. V. Frankland, V. M. Harik, G. M. Odegard, D. W. Brenner and T. S. Gates, Compos. Sci. Technol. 63, 1655 (2003).

48. J. Gou, B. Minaie, B. Wang, Z. Liang and C. Zhang, Comp. Mater. Sci. 31: 225 (2004).

49. C. Q. Ru, Phys. Rev. B 62, 9973 (2000).

50. C. Q. Ru, J. Mech. and Phys. Solids 49, 1265 (2001).

51. G. M. Odegard, T. S. Gates, L. M. Nicholson and K. E. Wise, Compos. Sci. Technol. 62, 1869 (2002).

52. P. Zhang, Y. Huang, P. H. Geubelle, P. A. Klein and K. C. Hwang, Int. J. Solids. Struct. 39, 3893 (2002).

53. H. Jiang, P. Zhang, B. Liu, Y. Huang, P. H. Geubelle, H. Gao and K. C. Hwang, K.C., Comp. Mater. Sci. 28, 429 (2003).

54. E. T. Thostenson and T. W. Chou, J. Phys. D-Appl. Phys. 36, 573 (2003).

55. A. Needleman, J. Appl. Mech. 54, 525 (1987).

56. G. T. Camacho and M. Ortiz, Int. J. Solids Struct. 33, 2899 (1996).

57. P. H. Geubelle and J. S. Baylor, Compos. B 29, 589 (1998).

58. Y. Huang and H. Gao, J. Appl. Mech. 68, 169 (2001).

59. P. Zhang, P. A. Klein, Y. Huang, H. Gao, H. and P.D. Wu, Comput. Modeling Eng. Sci. 3, 263 (2002).

60. D. V. Kubair, P. H. Geubelle and Y. Huang, J. Mech. Phys. Solids 50, 1547 (2002).

61. D. V. Kubair, P. H. Geubelle and Y. Huang, Eng. Fract. Mech. 70, 685 (2003).

62. O. Samudrala, Y. Huang and A. J. Rosakis, J. Mech. Phys. Solids 50, 1231 (2002).
63. O. Samudrala and A. J. Rosakis, Eng. Fract. Mech. 70, 309 (2003).

64. G. Thiagarajan, K. J. Hsia and Y. Huang, Eng. Fract. Mech. 71, 401 (2004).

65. G. Thiagarajan, Y. Huang and K. J. Hsia, J. Appl. Mech. 71, 796 (2004).

66. H. Tan, Y. Huang, C. Liu and P. H. Geubelle, Int. J. Plasticity 21, 1890 (2005).

67. H. Tan, C. Liu, Y. Huang and P. H. Geubelle, J. Mech. Phys. Solids 53, 1892 (2005).

68. H. Tan, C. Liu, Y. Huang and P. H. Geubelle, Int. J. Multiscale Comput. Eng. 4(1), 147 (2006).

69. V. C. Li, C. M. Chan and K. Y. Leung, Cement Concrete Res. 17, 441 (1987).

70. Z. K. Guo, A. S. Kobayashi, J. C. Hay and K. W. White, Eng. Fract. Mech. 63, 115 (1999).

71. I. Mohammed and K. M. Liechti, J. Mech. Phys. Solids 48, 735 (2000).

72. Z. P. Bazant, Eng. Fract. Mech. 69, 165 (2002).

73. M. Elices, G. V. Guinea, J. Gomez and J. Planas, Eng. Fract. Mech. 69, 137 (2002).

74. S. S. Hong and K. S. Kim, J. Mech. Phys. Solids 51, 1267 (2003).

75. L. Y. Jiang, Y. Huang, H. Jiang, G. Ravichandran, H. Gao, K. C. Kwang and B. Liu, J. Mech. Phys. Solids 54, 2436 (2006).

76. W. B. Lu, J. Wu, L. Y. Jiang, Y. Huang, K C Hwang and B. Liu, Phil. Magz. (in press, 2007).

77. H. Tan, L. Y. Jiang, Y. Huang, B. Liu and K. C. Hwang, Compos. Sci. Technol. (in press, 2007).

78. L. A. Girifalco, M. Hodak and R. S. Lee, Phys. Rev. B 62, 13104 (2000).

79. D. C. Rapaport, Molecular Dynamics Simulation, Cambridge University Press, Cambridge (2004)

80. Y. Huang, J. Wu and K. C. Hwang, Phys. Rev. B 74, 245413 (2006). 